\title{
Effect of Table Tennis Trainings on Biomotor Capacities in Boys
}

\author{
Murat Taş \\ Manisa Celal Bayar University, Manisa, Turkey
}

\begin{abstract}
The aim of this study is to investigate whether the biomotor capacities of boys doing table tennis trainings are affected. A total of 40 students, as randomly selected 20 test groups and 20 control groups at an age range of 10-12 participated in the research. Statistical analysis of data was performed using Statistic Package for Social Science (SPSS) 20.0 statistical package program. Mann Whitney $U$ Test was used in comparison of two variable groups, and the result being $p<0.05$ was considered significant when examined statistically. In pre-test and final-test comparisons of the subjects, it was detected that the statistical differences between flexibility, vertical jump, standing long jump, $30 \mathrm{~m}$ sprint, anaerobic strength, maximal oxygen capacity (MaxVO2) right and left hand grip strength, visual and audio reaction times, resting heart rate, and forced expiration volume (FEV) and FEV1 measurements were significant in favor of the test group $(p<0.05)$, while there was no statistically significant differences between the two groups in height, body weight, body fat percentage, body mass index, systolic and diastolic blood pressure, and vital capacity (VC) and forced vital capacity (FVC) values $(p>0.05)$. At the end of this study, it was observed that table tennis trainings provided positive contributions to biomotor capacities in boys of the group aged 10-12.
\end{abstract}

Keywords: boy, table tennis, training, biomotor capacities

\section{Introduction}

Child is a being, which constantly moves, and is completely active. He is in constant motion particularly after he starts walking. Child needs movement in order to function his organs, enhance his skeletal structure, develop his lungs, activate his blood, and strengthen his nerve-muscle connections (Yarımkaya \& Ulucan, 2015).

Human is in mobility within the environment he is in while continuing his development as a live being. Movement is expressed stated that human organism is created for movement. Movement is expressed as change in any part of body or in full body position (Saygin, Polat, \& Karacabey, 2005).

Movement training programs to be implied must be able to develop the physical suitability and perceptual socio-emotional properties of child (Watts, Joubert, Lish, Mats, \& Wilkins, 2003).

Table tennis improves concentration, reaction speed and coordination, arm and body muscles, and respiratory and circulatory functions; it also contributes to development of hand-eye coordination, timing, and balance functions (Ăggön \& Ağırbaş, 2015).

This study was conducted in order to examine the effect of table tennis trainings, which were applied for 16 weeks, on biomotor capacities in boys aged 10-12.

Murat Taş, Dr., associate professor, Faculty of Sport Sciences, Manisa Celal Bayar University. 


\section{Materials and Method}

Forty (20 test and 20 control groups) boys selected randomly within the age range of 10-12 studying in Bitlis Tatvan Fatih Secondary School participated in the research. Table tennis trainings were applied one hour for four days a week for 16 weeks on the test group, while no training was applied on the control group. Measurements in the research were performed by taking results of the pre-test as well as the final test at the end of 16 weeks.

\section{Tests Applied}

Height and body weight measurement. Body weight of the subjects was measured barefoot in a height measuring scale with a sensitivity of up to 20 grams (Desis M301 brand). Their weights were again measured by adjusting the height test gauge on the scale such that it touched their heads (Zorba \& Ziyagil, 1995).

Heart rate. Subjects were rested for 15 minutes before measuring heart rate. Heart rate was measured with the sphygmomanometer (Omron M2) attached to the left arms of the subjects (Günay, Tamer, \& Cicioğlu, 2005).

Systolic and diastolic pressure. Subjects were rested for 15 minutes before measuring systolic and diastolic pressure. Systolic and diastolic pressure were measured with the sphygmomanometer (Omron M2) attached to the left arms of the subjects (Günay et al., 2005).

Vertical jumping. A measurement stand was placed on the wall in a manner accessible by the subjects. Subjects tried to reach out to this measurement stand as much as they could with their feet contacting the ground, and later both the highest distance they could reach by jumping and this distance were measured. The difference between the height, which was reached by standing, and the height, which was reached by jumping, constituted the test result. Subjects were applied the test twice and their best grade was recorded (Kamar, 2003).

Anaerobic strength. Anaerobic strength of the subjects was measured through the Sayers Formula ( $p=$ $60.7 \times$ vertical jump distance $+45.3 \times$ body weight) (Günay et al., 2005).

Thirty-meter sprint. Subjects ran at a speed of $30 \mathrm{~m}$ (standing) on a scaled ground with the start command, and the best of the two assessments was recorded (Hoare, 2000).

Hand grip strength. Right and left grip strengths of the subjects were measured with digital hand dynamometer. Subjects were applied the test twice and their best grade was recorded (Günay et al., 2005).

Body mass index. Body mass index of the subjects was measured with Bodystat 1,500 body analysers. Age, height, and weight information were entered in the analyser while the subjects laid back. Measurements were taken from their right hand and right foot with the aid of an electro band.

Body fat percentage. Body fat percentage of the subjects was measured with Bodystat 1,500 body analyserds. Age, height, and weight information were entered. Measurements were taken by adhering electro bands to the right hand and right foot of the subjects while they laid back.

Standing long jump. Start point was determined and starting line was drawn in order for the subjects to jump. Meter was opened next to the starting line in jumping direction of the subjects and adhered to the ground via bands. The distance they jumped as well as the distance between the jumping line and heel were measured. Best of the two assessments was recorded (Kamar, 2003).

Flexibility. Sit and reach test was used in measurement of flexibility. Test stand was $35 \mathrm{~cm}$ long, $45 \mathrm{~cm}$ wide, and $32 \mathrm{~cm}$ high. Upper surface length of the stand was $45 \mathrm{~cm}$ and its width was $45 \mathrm{~cm}$. Upper surface was outside by $15 \mathrm{~cm}$ more than the surface its feet leaned on $0-50 \mathrm{~cm}$ measurement grid was determined with parallel line intervals of $5 \mathrm{~cm}$ of each on the upper surface. Subjects sat on the ground and leaned their bare sole 
straight on the test stand. They slowly pushed the grid forward by bending their body forward and reaching forward as much as they could without bending their knees and keeping their hands in front of the body. They waited for 1-2 seconds at the furthest point without stretching forward or backwards. Test was repeated twice and the highest value was recorded (Günay et al., 2005).

Reaction measurement. Audio and visual reaction times of the subjects were detected using Newtest 1000 device. In measuring reaction times, attention was paid to the fact that measurement place was free of noise and received light. Two measurements were taken from each subject following a trial against light and sound warnings. Best value of the two measurements was recorded in milliseconds. Dominant hands of the subjects were used in measuring audio and visual reaction times (Tamer, 2000).

Spirometric measurements. Respiratory measurements of the subjects were performed with SpirolabII spirometer. Subjects were taken to test in rested condition. Vital capacity (VC), forced vital capacity (FVC), forced expiration volume in one second (FEV1), and forced expiration volume percentages in one second (FEV1\%) of the subjects were determined (Günay et al., 2005).

Maximal oxygen capacity (MaxVO2) measurement. Twenty-meter pull-up run test was applied for MaxVO2 measurement. Twenty-meter pull-up run test cassette in compliance with the protocol was used in order to determine running speed. Subjects ran in a $20 \mathrm{~m}$ track by touching the determined lines by their feet. The test was continued until the subjects made two errors. Test result was recorded as $\mathrm{ml} / \mathrm{kg} / \mathrm{min}$.

Fernhall Formula $(0.35 \times$ shuttle number $-0.59 \times$ V.K.I. $-4.61 \times(1)+50.6)$ was used in order to transform the total number of shuttle ran at the end of the shuttle run into MaxVO2 value (Tamer, 2000).

Table 1

Sixteen Week Table Tennis Training Program Applied (Source: Asan, 2011)

\begin{tabular}{lllll}
\hline Week & Work hour & Work place & Number of student & Subject to be handled \\
\hline 1 & $14.30-15.30$ & Table Tennis Hall & 20 & Introduction to table and paddle-free moves with ball \\
2 & $14.30-15.30$ & Table Tennis Hall & 20 & Paddle holding and dribbling with paddle \\
3 & $14.30-15.30$ & Table Tennis Hall & 20 & Dribbling with paddle \\
4 & $14.30-15.30$ & Table Tennis Hall & 20 & Paddle-free forehand serving at table \\
5 & $14.30-15.30$ & Table Tennis Hall & 20 & Forehand service stroke with paddle (flat service) \\
6 & $14.30-15.30$ & Table Tennis Hall & 20 & Backhand service stroke with paddle (reverse service) \\
7 & $14.30-15.30$ & Table Tennis Hall & 20 & Forehand drive (flat ball strokes) \\
8 & $14.30-15.30$ & Table Tennis Hall & 20 & Forehand -backhand drive (flat-reverse stroke) \\
9 & $14.30-15.30$ & Table Tennis Hall & 20 & Forehand -backhand drive (flat-reverse stroke) \\
10 & $14.30-15.30$ & Table Tennis Hall & 20 & Smash. (forehand and backhand stroke) \\
11 & $14.30-15.30$ & Table Tennis Hall & 20 & Chop. (interrupted strokes, forehand) \\
12 & $14.30-15.30$ & Table Tennis Hall & 20 & Flip (ball stroke workouts) \\
13 & $14.30-15.30$ & Table Tennis Hall & 20 & Drop (short ball workouts) \\
14 & $14.30-15.30$ & Table Tennis Hall & 20 & Spin (spin strokes) \\
15 & $14.30-15.30$ & Table Tennis Hall & 20 & Spin (spin strokes) \\
16 & $14.30-15.30$ & Table Tennis Hall & 20 & Match games \\
\hline
\end{tabular}

Note. Warm-up: 15 minutes; main training: 45 minutes; and cool down: 5 minutes.

\section{Table Tennis Training Program Applied}

Intensive interval training was used in our study, and training intensity was followed as $65 \%$. Fluid (water) intake was allowed according to individual requirement throughout the 16-week training period (see Table 1). 


\section{Data Analysis}

Statistical analysis of data was performed using Statistic Package for Social Science (SPSS) 20.0 statistical package program in this study. Mann Whitney $U$ Test was used in comparison of two variable groups, and the result being $p<0.05$ was considered significant when examined statistically.

\section{Findings}

When Table 2 is examined, no significant difference was observed between the two participant groups of the study in initial and final measurements of their height, body weight and body mass index values $(p>0.05)$.

Table 2

Comparison of the Physical Properties of Participants

\begin{tabular}{llccll}
\hline Variables & & Test group $(N=20)$ & Control group $(N=20)$ & $Z$ & $p$ \\
\hline \multirow{2}{*}{ Height $(\mathrm{cm})$} & Pre test & $1.44 \pm 0.64$ & $1.42 \pm 0.65$ & -0.0800 & 0.429 \\
& Post test & $1.46 \pm 0.63$ & $1.43 \pm 0.66$ & -1.239 & 0.221 \\
\multirow{2}{*}{ Body Weight $(\mathrm{kg})$} & Pre test & $37.86 \pm 7.93$ & $34.60 \pm 3.91$ & -1.177 & 0.242 \\
& Post test & $37.39 \pm 7.67$ & $35,22 \pm 4.25$ & -0.514 & 0.620 \\
V.Y.Y $(\%)$ & Pre test & $22.49 \pm 5.60$ & $24.17 \pm 5.47$ & -1.001 & 0.314 \\
& Post test & $21.13 \pm 4.66$ & $24.51 \pm 5.11$ & -1.731 & 0.086 \\
V.K.I $\left(\mathrm{kg} / \mathrm{m}^{2}\right)$ & Pre test & $18.31 \pm 3.42$ & $17.06 \pm 2.88$ & -0.961 & 0.341 \\
& Post test & $17.60 \pm 3.12$ & $17.54 \pm 3.09$ & -0.257 & 0.799 \\
\hline
\end{tabular}

Table 3

Comparison of Pre and Post Test Values of Participants

\begin{tabular}{llllll}
\hline Variables & & Test group $(N=20)$ & Control group $(N=20)$ & $Z$ & $p$ \\
\hline \multirow{2}{*}{ Flexibility (cm) } & Pre test & $16.25 \pm 6.10$ & $15.85 \pm 6.30$ & -0.327 & 0.758 \\
& Post test & $22.40 \pm 6.56$ & $15.55 \pm 6.21$ & -2.863 & $0.004^{*}$ \\
Vertical jumping (cm) & Pre test & $18.30 \pm 4.41$ & $16.57 \pm 4.77$ & -1.603 & 0.114 \\
& Post test & $23.15 \pm 5.06$ & $16.65 \pm 4.46$ & -3.675 & $0.000^{*}$ \\
Standing long jump (cm) & Pre test & $114.7 \pm 16.2$ & $108.8 \pm 16.0$ & -1.029 & 0.314 \\
& Post test & $130.6 \pm 14.9$ & $104.4 \pm 15.9$ & -4.146 & $0.000^{*}$ \\
$30 \mathrm{~m}$ (sec.) & Pre test & $5.76 \pm 0.559$ & $5.69 \pm 0.324$ & -0.135 & 0.904 \\
& Post test & $5.37 \pm 0.306$ & $5.79 \pm 0.349$ & -3.367 & $0.001^{*}$ \\
Anaerobic strength (W) & Pre test & $2,826 \pm 382$ & $2,573 \pm 336$ & $-2,340$ & $0.018^{*}$ \\
& Post test & $3,099 \pm 383$ & $2,563 \pm 327$ & $-3,895$ & $0.000^{*}$ \\
MaxVO2 (ml/kg/min.) & Pre test & $47.13 \pm 5.19$ & $46.26 \pm 3.72$ & -0.784 & 0.445 \\
& Post test & $48.83 \pm 5.60$ & $45.19 \pm 3.55$ & -2.380 & $0.017^{*}$ \\
\hline
\end{tabular}

Note. ${ }^{*} p<0.05$.

While no significant difference was observed in initial measurements between the two groups in flexibility, vertical jump, standing long jump, $30 \mathrm{~m}$ sprint, and MaxVO2 values of the participant test and control group boys $(p>0.05)$, a significant difference in favor of the test group was observed in their final measurements $(p>$ 0.05) (see Table 3). Significant difference in favor of the test group was observed in initial and final measurement in anaerobic strength values $(p>0.05)$.

When Table 4 is examined, a significant difference in favor of the test group was found in comparison of initial and final measurements of the right and left hand grip values of the participant test and control group 
boys $(p>0.05)$. While a significant difference occurred in favor of the control group in the initial measurement of visual reaction values $(p<0.05)$, a significant difference was found in favor of the test group in the final measurement $(p<0.05)$. While no significant differences were found between the two groups in initial measurements of audio reaction and perception values $(p>0.05)$, significant difference in favor of the test group was observed in the final measurement $(p>0.05)$. In systolic and diastolic blood pressure values, no significant difference was observed between the two groups in comparison of initial and final measurements $(p>$ $0.05)$.

Table 4

Comparison of Pre and Post Test Values of Participants

\begin{tabular}{|c|c|c|c|c|c|}
\hline Variables & & Test group $(N=20)$ & Control group $(N=20)$ & $Z$ & $p$ \\
\hline \multirow{2}{*}{ Right hand grip strength (kg) } & Pre test & $16.76 \pm 4.74$ & $12.98 \pm 3.03$ & -2.923 & $0.003^{*}$ \\
\hline & Post test & $26.34 \pm 6.99$ & $12.96 \pm 3.17$ & -5.181 & $0.000^{*}$ \\
\hline \multirow{2}{*}{ Left hand grip strength (kg) } & Pre test & $15.76 \pm 4.92$ & $12.64 \pm 2.39$ & -2.463 & $0.013^{*}$ \\
\hline & Post test & $21.17 \pm 5.21$ & $12.40 \pm 2.22$ & -5.142 & $0.000^{*}$ \\
\hline \multirow{2}{*}{ Visual reaction (sec.) } & Pre test & $0.3370 \pm 0.088$ & $0.2840 \pm .066$ & -2.059 & $0.040^{*}$ \\
\hline & Post test & $0.1835 \pm 0.028$ & $0.3140 \pm .082$ & -5.147 & $0.000^{*}$ \\
\hline \multirow{2}{*}{ Audio reaction (sec.) } & Pre test & $0.2810 \pm 0.060$ & $0.2785 \pm .070$ & -0.353 & 0.738 \\
\hline & Post test & $0.1535 \pm 0.015$ & $0.2905 \pm .063$ & -5.436 & $0.000^{*}$ \\
\hline \multirow{2}{*}{ RHR (pulse/min.) } & Pre test & $94.50 \pm 9.58$ & $92.10 \pm 10.0$ & -0.937 & 0.355 \\
\hline & Post test & $85.25 \pm 8.46$ & $94.30 \pm 10.8$ & -3.046 & $0.002^{*}$ \\
\hline \multirow{2}{*}{ Blood pressure systolic (mm/Hg) } & Pre test & $108.5 \pm 21.0$ & $109.5 \pm 11.9$ & 0.000 & 1.000 \\
\hline & Post test & $118.0 \pm 17.3$ & $122.0 \pm 13.9$ & -0.578 & 0.583 \\
\hline \multirow{2}{*}{ Blood pressure diastolic $(\mathrm{mm} / \mathrm{Hg})$} & Pre test & $73.00 \pm 13.4$ & $73.50 \pm 9.88$ & -0.212 & 0.841 \\
\hline & Post test & $75.00 \pm 7.60$ & $70.00 \pm 6.48$ & -2.042 & 0.068 \\
\hline
\end{tabular}

Note. ${ }^{*} p<0.05$.

Table 5

Comparison of Pre and Post Test Values of Participants

\begin{tabular}{llllll}
\hline Variables & & Test group $(N=20)$ & Control group $(N=20)$ & $Z$ & $p$ \\
\hline \multirow{2}{*}{ VC (lt) } & Pre test & $2.278 \pm 0.933$ & $2.561 \pm 0.522$ & -1.502 & 0.134 \\
& Post test & $2.784 \pm 0.991$ & $2.341 \pm 0.515$ & -1.381 & 0.174 \\
FVC (lt) & Pre test & $1.700 \pm 0.555$ & $1.762 \pm 0.604$ & -0.081 & 0.947 \\
& Post test & $2.143 \pm 0.805$ & $1.757 \pm 0.421$ & -1.747 & 0.081 \\
FEV (lt) & Pre test & $1.724 \pm 0.557$ & $1.668 \pm 0.439$ & -0.311 & 0.756 \\
& Post test & $2.172 \pm 0.788$ & $1.657 \pm 0.392$ & -2.666 & $0.007^{*}$ \\
FEV1 (lt) & Pre test & $91.32 \pm 0.457$ & $88.19 \pm 0.467$ & -2.152 & $0.030^{*}$ \\
& Post test & $93.42 \pm 0.448$ & $87.61 \pm 0.494$ & -3.383 & $0.000^{*}$ \\
\hline
\end{tabular}

Note. ${ }^{*} p<0.05$.

According to the results in Table 5, no significant difference was observed between the participant test and control group boys in comparison of initial and final measurements of their VC and FVC values $(p>0.05)$. While no significant difference was observed between the test and control group in initial measurements of FEV values $(p>0.05)$, a significant difference was observed in favor of the test group in their final measurement $(p>0.05)$. A significant difference was observed between the two groups in initial and final measurements of FEV1 values $(p>0.05)$. 


\section{Discussions and Conclusions}

No significant difference was observed in height, body weight, body fat percentage, and body mass index values of the male test and control group in their pre and post-study measurements. Despite the fact that height increase is considered to arise from developmental properties, reduction in body weight, body fat percentage, and body mass index of the test group boys can be associated with the trainings made.

When the studies on the body mass index are examined. In the study, they conducted on 177 children aged 12-14 in the province of Erzincan, Çolak, and Kaya (2006) calculated the body mass index average in 12 -year-old group males as $18.11 \pm 3.21 \mathrm{~kg} / \mathrm{m}^{2}$ for the provincial centre, while as $17.34 \pm 2.51 \mathrm{~kg} / \mathrm{m}^{2}$ for districts. The research results showed parallelism with this research.

In the research he conducted, Kavak (2006) obtained $17.4 \pm 1.8 \mathrm{~kg} / \mathrm{m}^{2}$ as the average value in 45 males of 10-year-old group. The same variant was detected as $17.6 \pm 1.6 \mathrm{~kg} / \mathrm{m}^{2}$ in 131 male subject of 11-year-old group. The same value was detected as $18.5 \pm 1.6 \mathrm{~kg} / \mathrm{m}^{2}$ in 152 male subject of 12 -year-old group. In the study, the final measurement values of the test group males were found as $17.60 \pm 3.12 \mathrm{~kg} / \mathrm{m}^{2}$. While no evident differences are observed in boys in values of all age groups, it can be said that the change in values arises from change in height and body weight. While there was a reduction in favor of the test group in initial and final measurements of the male test and control group in body fat percentage (V.Y.Y.) values, this reduction is not statistically significant.

Watts et al. (2003) have stated that mountaineers aged 11-12, who deal with various sports, have lower fat percentage than non-active children.

Adeniran and Torida (1988) detected fat reduction by $8.1 \%$ in male students of 13-17-year-old age group as a result of the 8-week continuous running training method they performed. This can be regarded as the indicator that workouts performed reduce body fat percentage.

While only significant difference was detected in anaerobic strength value in favor of the test group in the initial measurement between pre and post-study values of flexibility, vertical jump, standing long jump, $30 \mathrm{~m}$ sprint, anaerobic strength and MaxVO2 values, significant differences were detected in favor of the test group in all parameters in the final measurement.

In the study they performed, (Kerkez, Kalkavan, \& Öztürk, 2001) reported the flexibility of 11-year-old children as $13.73 \mathrm{~cm}$. In the study, he performed, Yenal (1996) detected significant differences in 10-11-year-old boys in the test group compared to the control group in terms of flexibility. Saygin (2003) had reported significant difference in terms of flexibility between children with mild level of activity and children with medium level of activity. It can be said that the duration, severity and intensity of the workouts performed affect flexibility level.

In the study, the flexibility value of the male test group in the final measurement was found as $22.40 \pm$ $6.56 \mathrm{~cm}$. Data obtained in this study show parallelism with the above-mentioned study in terms of increase in flexibility values.

Katie et al. (2003) found differences in vertical jump values of the children receiving sport training compared to the children who do not receive sport training. Saygin (2003) had found significant difference in terms of vertical jump between children with mild level of activity and children with medium level of activity.

In the study, the vertical jump distance of the test group females was found as $19.55 \pm 5.44 \mathrm{~cm}$, while of the males as $23.15 \pm 5.06 \mathrm{~cm}$. The fact that the increase in vertical jump distance is in favor of the test group can be associated with the effect of the trainings performed. 
In their study, Pekel, Balc1, Pepe, Arslan, Bağc1, Tamer, and Aydos (2004) reported standing long jump test results of 11-13-year-old children as $181.2 \pm 16.2 \mathrm{~cm}$ for boys. In the study they performed on trained children. Karabulak and Kılınç (2006) stated standing long jump as $206.40 \pm 18.59 \mathrm{~cm}$. Difference is seen between our finding $(130.6 \pm 14.9 \mathrm{~cm})$ and the findings of other researches. The reason for this may arise from the fact that children are in developmental age as well as the difference of sport ages and the content of the trainings they do.

When the studies on $30 \mathrm{~m}$ sprint are examined; while Gül, Seyrek, and Sugurtin (2006) found the average value as $5.99 \pm 0.53 \mathrm{sec}$., they measured the minimum value as $4.84 \mathrm{sec}$. and the maximum value as $9.13 \mathrm{sec}$. in the research they performed on 84 boys aged 10-12, who did not do sports. Kien and Chiodo (2003) had reported that 10-12-year-old secondary school children, who participate in recreational programs, are stronger than the children of their own age group, who do not participate in recreative sport activities.

Significant differences were found in 20,30 and $40 \mathrm{~m}$ sprint values as a result of the exercise applied three days a week on children aged 10-12 (Diallo, Dore, Duche, \& Van, 2001). When the studies $30 \mathrm{~m}$ sprint variable can be associated with the increases in strength parameters, increase of the anaerobic strength, and the workouts performed.

Saygin (2003) had found significant difference in terms of anaerobic strength between children with mild level of activity and children with medium level of activity. Kien and Chiodo (2003) had reported that 10-12-year-old secondary school children, who participate in recreational programs, are stronger than the children of their own age group, who do not participate in recreative sport activities.

It can be said that through the influence of the workouts performed, vertical jump and height increase values also increase the anaerobic strength value.

Erol, Cicioğlu, and Pulur (1999) found significant development in terms of MaxVO2 in the test group compared to the control group as a result of 10-week regular exercise. In another study, significant differences were detected in MaxVO2 parameters as a result of the interval training, which was performed on 10-12-year-old children as 100-800 $\mathrm{m}$ and for 10 to 30 minutes with an intensity of $70-80 \%$ (Mahen \& Voccaro 1999). The workouts performed can be said to have increased use of oxygen capacity in children.

Significant differences in favor of the test group occurred in initial and final measurements of pre and post-left and right hand grip strength values of the male test and control group. While there was a significant difference in favor of the test group in the initial measurement of the visual reaction time, this difference was significant in favor of the test group in pre-study measurement. While there was no significant difference between the test and control group in the initial measurement of audio reaction and rest heart rate values, significant differences occurred in favor of the test group in the final measurement. No significant difference occurred between the test and control group in initial and final measurements of systolic and diastolic blood pressure values. While no significant differences occurred between the two groups in initial measurements of pre and post-study of right and left hand grip strength, audio and video reaction times, and resting heart rates of the female test and control group, significant differences in favor of the test group occurred in the final measurements performed after the study. No significant difference occurred between the test and control group in initial and final measurements of systolic and diastolic blood pressure values.

Katie, Brad, Joanne, Linda, and Terence (2003) had found difference in terms of grip strength between the primary school children, who dominantly receive table tennis training, and the children who receive sport training and table tennis training in a balanced manner. 
Ziyagil, Tamer, Zorba, Uzuncan, and Uzuncan (1996) had detected that 11-year-old athletes had significant differences compared to non-athletes in terms of their grip strengths. One of the properties of the table tennis branch is that very fast strikes can be made with a strong grip and arm strength. Because of this property, it is seen that table tennis trainings improve grip strength. As a result of the research, it is considered that grip strengths of the boys in the test group developed positively.

In their study, they performed in order to determine to what degree the hand-eye coordination and reaction times of first graders are affected by folk dance study, which is performed for one lesson hour once week for 12 weeks, Yetkin and Kayapınar (2008) reported that they had found significant results in initial-final test values in the test group.

Reaction time was examined in children aged 8-13, who had previously participated in sportive activities and who had not, and the reaction time was detected to be shorter in the group that had participated in sportive activities Montes, Bueno, Candel, and Pans (2000). In their study, Mouelhi, Bouzaouach, Tenenbaum, Ben, Feki, and Bouaziz (2006) reported that the respond given to external environmental warnings can be accelerated through exercise.

It can be said that table tennis trainings improve the reaction time of boys.

In a research conducted on resting heart rate, heart rate between ages 11-12 was determined as 82-84 pulses/minute. Children have higher minute volume compared to adults. Blood circulation process is shorter at these ages. Blood is delivered to the cells in child organism faster as a result of the physical movements performed (Coşan, 1992).

In our study final measurement values of the male test group were found as $85.25 \pm 8.46$ beats/minute. It can be expressed that the values obtained in this study are similar to the values obtained in the aforesaid study performed. Heart rate following load reduces gradually due to advanced age and training developments. In children, heart rate with the effect of training is quite below the average of children who do not do sport. Heart rate returns to normal following loading quicker in trained children compared to untrained children (Muratl1, 2001).

The reason for reduction observed in resting heart rate values of the test group children is considered to be due to the increase in pulse volume which formed with the effect of training.

According to a research performed on systolic and diastolic blood pressure, children performing physical activities had lower blood pressure than those children who do not perform physical activities (Kerkez, 2001). When the results of our study are compared to the literature results, they are partially similar. Dekkers, Snieder, Van, and Treiber (2002) examined the effect of height, gender, body fat, and socio-economic status on development of tension within a period of 10 years. In the research performed on 745 children, it was found that socio-economic status caused difference in height, body weight and body fat levels. Detection of the mechanisms responsible for increase of tension in childhood will help prevention of hypertension in adulthood. It can be said that the blood pressure values in this study are within the normal values in the literature.

No significant difference was found between the two groups in initial and final measurements of pre and post VC and FVC values of the test, and control group. While no significant difference was observed between the test and control group in initial measurements of FEV values, a significant difference was observed in favor of the test group in their final measurement. A significant difference was observed in favor of the test group in initial and final measurements of FEV1 values. Significant differences in favor of the test group were found in initial and final measurements of pre and post $\mathrm{VC}$ values of the female test and control group. While no 
significant differences were found between the two groups in initial measurements of FVC, FEV and FEV1 values, significant differences in favor of the test group formed in their final measurements.

Vijayan, Reetha, Kuppurao, Venkatesan, and Thilakavathy (2000) conducted lung functional test in 469 children aged 7-19. FVC and FEV1 correlations were generally the highest regarding height, followed by weight and age. While height affected the equation substantially in boys, age and weight were more effective in girls.

Aim of the study performed by Shephard and Lavallee (1996) was to test the effect of enriched physical education program on static and dynamic lung volume growth and development of primary school children. 546 students aged 7-12 attended an additional 5 hours of physical education lesson every week. They reported that a regular physical education program can increase lung volume (FEV1 and FVC) in primary school children. In the study, they performed on 11-14-year-old groups of children who do and do not sport, Alpay, Altuğ, and Hazar (2008) reported that vital and force vital capacity were high in the group that does sport.

The fact that vital capacity values showed increase in favor of the test group children is considered to have derived from the positive effect of training on respiratory system.

As a consequence; development was seen both in the test and the control group due to the fact that children are in developmental age; however, a significant difference was detected in favor of the test group boys in terms of biomotor capacities. This difference can be said to arise from the effect of training, physical development of individuals, or hormonal change that occurs due to sexual maturity. It is considered that correct training practices to be performed will contribute to growth and development.

\section{References}

Adeniran, S. A., \& Torida, A. L. (1988). Effects of different running programmers on body fat and blood pressure in schoolboys aged 13-17 years. The Journal of Sports Medicine and Physical Fitness, 28(3), 69-79.

Ağgön, E., \& Ağırbaş, Ö. (2015). İnönü University. Journal of Physical Education and Sport Sciences, 2(2), 12-20.

Alpay, B., Altuğ, K., \& Hazar, S. (2008). Evaluation of certain respiratory and circulatory parameters of 11-13-year-old group students playing in primary school teams through comparison with students who do not do sports. Mehmet AkifErsoy Üniversity, Journal of Education Faculty, 8(17), 22-29.

Asan, R. (2011). Effect on eight-week table tennis exercise on attention in 9-13 year-old children (Master's thesis, Selçuk University, Konya, Turkey).

Aslan, A. (2006, October 27-29). Comparison of several performance and anthropometric properties in trained children. In Seventh Sport Sciences Congress Seminar Book, Antalya, Turkey.

Çolak, M., \& Kaya, M. (2006). Evaluation of body mass index and skinfold parameter of 12-14-year-old children living in the province of Erzincan. Gazi University, Journal of Physical Education and Sport Sciences, 11(4), 23-30.

Coşan, F., \& Demir, A. (1992). Physical conformity norms of Turkish children, Istanbul Olympic Games Preparation and Organization Committee Educational Publications. İstanbul, Turkey: Mart Matbaac1lı.

Dekkers, J. C., Snieder, H., Van, E. J., \& Treiber, F. A. (2002). Moderators of blood pressure development from childhood to adulthood: A 10-year longitudinal study. Journal of Pediatrics, 14(6), 770-779.

Diallo, O., Dore, E., Duche, P., \& Van Praagh, E. (2001). Effects of plyometric training followed by a reduced training programme on physical performance in prepubescent soccer players. Journal of Sports Medicine and Physical Fitness, 41(3), 342-348.

Erol, E., Cicioğlu, İ., \& Pulur, A. (1999). Effects of endurance training towards 13-14-year-old male basketball players on body composition and certain physical, physiological and blood parameters. Journal of Physical Education and Sport Sciences, 4 , $12-20$.

Gül, G. K., Seyrek, E., \& Sugurtin, M. (2006, November 3-5). 10-12 Comparison of certain anthropometric properties between boys aged 10-12 who receive and do not receive basic athletics sport training. In Ninth International Sport Sciences Congress, Muğla, Turkey. 
Günay, M., Tamer, K., \& Cicioğlu, İ. (2005). Sport physiology and performance measurement. Ankara, Turkey: GaziKitapevi.

Hoare, D. G. (2000). Predicting success in junior elite basketball players the contribution of anthropometric and physiological attributes. Journal of Science \& Medicine, 34, 391-405.

Kamar, A. (2003). Skill and performance tests in sport. Ankara, Turkey: Nobel YayınDağıtım.

Karabulak, A., \& Kilınç, F. (2006). 12-14 years old male soccer players applied to the investigation of the effect of performance of combined training. Journal of Current Researches on Social Sciences, 6(2), 79-95.

Katie, M. M., Brad, S. M., Joanne, K, Linda, D. V., \& Terence, J. W. (2003). Contribution of timetabled physical education to total physical activity in primary school children cross sectional study. Thebmj (BMJ), 327( 592).

Karabulak, A., \& Kılınç, F. (2006). 12-14 years old male soccer players applied to the investigation of the effect of performance of combined training. Journal of Current Researches on Social Sciences, 6(2), 79-95.

Kavak, V. (2006). The determination of subcutaneous body fat percentage by measuring skinfold thickness in teenagers in Turkey. International Journal of Sport Nutrition and Exercise Metabolism, 16(3) 296-304. https://doi.org/10.1123/ijsnem.16.3.296

Kerkez, F., Kalkavan, A., \& Öztürk, A. (2001). Investigation of the effect of certain psychomotor and physical properties on coordinative skill in 9-11-year-old group boys from Van. Journal of Sport Researches, 5(1), 19-27.

Kien, C. L., \& Chiodo, A. R. (2003). Physical activity in middle school-aged children participating in a school-based recreation program. Archives of Pediatrics and Adolescent Medicine, 157(8), 811-815.

Montes, M. R., Bueno, I., Candel, J., \& Pans, A. M. (2000). Eye hand and eye-foot visual reaction times of young soccer players. Optometry, 71(12), 775-80.

Mouelhi, G. S., Bouzaouach, I., Tenenbaum, G., Ben, K. A., Feki, Y., \& Bouaziz, M. (2006). Simple and choice reaction times under varying levels of physical load in high skilled fencers. Journal of Sports Medicine and Physical Fitness, 46(2), 344-351.

Muratlı, S. (2001). Child and sport. Nobel YayınDă̆ıtım.

Pekel, H. A., Balcı, S. Ş., Pepe, H., Arslan, Ö., Bağc1, E., Tamer, K., \& Aydos, L. (2004). Evaluation of certain anthropometric properties and physical conformity parameters related to performance of children doing athletics. Eighth International Conference of Sport Sciences, Antalya.

Saygın, Ö. (2003). Examination of physical activity levels and physical conformities of 10-12-year-old children (Doctorate thesis, İstanbul, Turkey).

Saygın,Ö., Polat, Y., \& Karacabey, K., (2005). Effect of movement training on physical conformity properties in children. Firat University Medical Journal of Healths Sciences, 19(3), 205-212.

Shephard, R. J., \& Lavallee, H. (1996). Effects of enhanced physical education on lung volumes of primary school children. Journal of Sports Medicine and Physical Fitness, 36(3), 186-194.

Tamer, K. (2000). Measurement and evaluation of physical-physiological performance in sport. Ankara, Turkey: Bağırgan Yayımevi.

Vijayan, V. K., Reetha, A. M., Kuppurao, K. V., Venkatesan, P., \& Thilakavathy, S. (2000). Pulmonary function in normal South Indian children aged 7 to 19 years. Indian Journal of Chest Disease and Allied Science, 42(3), 147-156.

Watts, P. B, Joubert, L. M., Lish, A. K., Mats, J. D., \& Wilkins, B. (2003). Anthropometry of young competitive sport rock climbers. British Journal of Sports Medicine, 37(5), 420-424.

Yarımkaya, E., \& Ulucan, H. (2015). The effect of movement education program on the motor development of children. International Journal of New Trends in Arts, Sports \& Science Education, 4(1), 37-48.

Yenal, T. H. (1996). Effect of physical education and sport activities on motor skill and talent developments in primary school second term students (Doctorate thesis, İzmir, Turkey).

Yetkin, M. K., Kayapınar, F. Ç. (2008). Evaluation of the effect of twelve-week folk dance education on hand-eye coordination and reaction times primary school first grade students. Atatürk University Physical Education and Sport Sciences Journal, 10(4), 60-70.

Ziyagil, M. A., Tamer, K., Zorba, E., Uzuncan, S., \& Uzuncan, H. (1996). Evaluation of physical conformity and anthropometric properties of 10-12-year-old primary school students by age groups and their sport habits. Journal of Physical Education and Sport Sciences, 1, 20-28.

Zorba, E., \& Ziyagil, M. A. (1995). Body composition and measurement methods. Ankara, Turkey: Gen Matbaac1l1k Reklamc1l1k Ltd. Şti.. 